\title{
Editorial
}

\section{Combined immunodeficiency associated with DOCK8 mutations and related immunodeficiencies}

\author{
Helen C. Su* \\ Laboratory of Host Defenses, National Institute of Allergy and Infectious Diseases, National Institutes of Health, \\ Bethesda, MD, USA
}

\section{Introduction}

Studying rare inherited immune disorders in patients has led to improved understanding of how the human immune system is fundamentally regulated. This knowledge is needed to develop new therapeutic agents for immunodeficiency, autoimmunity, cancer, and transplantation. One such illustrative disorder is DOCK8 deficiency, whose molecular etiology was discovered only in the past year. The discovery of autosomal recessive $D O C K 8$ mutations and the identification of associated biomarkers have identified new areas of research and have also provided insights into similar and potentially related disorders, including the pathogenesis of certain associated viral infections. These developments are discussed in the eight reviews that are brought together in this special issue of Disease Markers.

In the first review, Alexandra Freeman and Steven Holland compare and contrast the clinical features of hyper-IgE syndrome due to STAT3, TYK2, and DOCK8 mutations. These three conditions were originally grouped as a single disease entity based primarily upon shared clinical findings of elevated serum IgE, eczema,

* Corresponding author: Dr. Helen Su, Building 10CRC, Room 5W3940, 10CRC Center Dr., MSC 1456, Bethesda, MD 20892-1456, USA. Fax: +1 301480 0983; E-mail: hsu@niaid.nih.gov. and susceptibility to infections. Most cases of hyperIgE syndrome result from autosomal dominant STAT3 mutations. By contrast, DOCK 8 mutations and the single reported case of $T Y K 2$ mutations are responsible for what was termed autosomal recessive hyper-IgE syndrome. As described by the authors, a number of clinical differences between DOCK8 deficiency and other forms of hyper-IgE syndrome exist, which suggest different disease mechanisms.

These clinical differences, as well as the associated genetic and laboratory biomarkers in DOCK8 deficiency, are described further in the second review by Qian Zhang and colleagues. The authors discuss how laboratory biomarkers and other experimental evidence point to specific mechanisms involving lymphocytes, which likely contribute to the disease manifestations. The demonstrated defects involve both CD8 T cells and $\mathrm{B}$ cell antibody responses, which are normally important for controlling a variety of infections including the chronic viral infections that are featured in this disease. Thus, the authors conclude that this disease can also be viewed as a combined immunodeficiency rather than a form of hyper-IgE syndrome.

The impaired antibody responses are the main focus of the third review by Katrina Randall and colleagues. By performing experiments in the Dock8deficient mice that they generated, the authors established that Dock8 is important for antibody persistence and memory and then elucidated the responsible im- 
munological mechanisms in B cells. These results were reported at around the same time as the discovery of DOCK8-deficient patients, thereby potentially explaining the functional antibody abnormalities seen in the patients. As shown by this example, the development of an animal model of the human disease has made it possible to study the immunological mechanisms in greater detail than can be easily done using patients' cells. These mice will no doubt continue to be a useful tool for future studies of disease pathogenesis.

In the fourth review, Sean McGee and Talal Chatila return us to the DOCK8 deficient patients. They describe what is known regarding how DOCK8 and related DOCK molecules biochemically function. Based upon this available knowledge they suggest that DOCK8 deficiency likely involves cytoskeletal dysfunction. Because DOCK8 is an atypical guanine nucleotide exchange factor that is thought to activate the Rho GTPases CDC42 and RAC, DOCK8 combined immunodeficiency not surprisingly clinically resembles both the Wiskott-Aldrich syndrome (WAS) that impairs CDC42 signaling, and disease due to known RAC2 mutations.

These two related disorders are the subject of the next two reviews. Michael Blundell and colleagues describe the Wiskott-Aldrich syndrome, a well-characterized combined immunodeficiency that results from mutations that cause loss of WAS protein (WASp) expression, cytoskeletal dysfunction, and a range of immune abnormalities. The authors review in detail the biochemical characterization of how WASp signals, as well as the phenotypic variants of WAS, i.e., X-linked thrombocytopenia due to missense mutations and $\mathrm{X}$ linked neutropenia due to activating mutations. In the other review, Sung-Yun Pai and colleagues describe how RAC functions in the immune system. In particular, they describe two patients with dominant interfering mutations in $R A C 2$, who have a phenotype resembling leukocyte adhesion deficiency (LAD) with prominent phagocytic defects. Interestingly, the one patient who was tested had T lymphocyte abnormalities that are consistent with mouse models of Rac deficiency. Together, both reviews expand upon the concept that DOCK8 deficiency may be pathogenically related to diseases known to affect Rho GTPases or their downstream effectors.
Finally, the last two reviews discuss related diseases that, like DOCK8 deficiency, present with an unusual susceptibility to cutaneous human papillomavirus infections. Françoise Bachelerie describes the well-characterized WHIM syndrome, which also features low serum immunoglobulins besides warts, and which usually results from activating mutations in the chemokine receptor CXCR4. Dysregulation of this CXCL12-CXCR4 signaling pathway is reviewed in detail, including the biochemical and biological consequences such as abnormal leukocyte trafficking. Interestingly, various DOCK molecules are known to play a role in cell trafficking, so a similar abnormality might explain the selective susceptibility to skin infections shared by WHIM and DOCK8 deficiency. In the other review, Tejas Patel and colleagues describe epidermodysplasia verruciformis, which presents with an isolated susceptibility to cutaneous human papillomavirus infections due to mutations in EVERI or EVER2. By contrast to various immunodeficiencies that can lead to a similar picture, the example of epidermodysplasia verruciformis is one in which disease results from abnormalities of non-immune cells within the skin. Whether or not DOCK8 serves a similar function in non-immune cells within the skin, thereby impacting the viral life cycle and accounting for the targeting of viral infections to the skin, warrants future investigations.

In summary, although DOCK8 immunodeficiency was only discovered in 2009 , this fascinating human disease has already provided insight into the important role of DOCK8 in regulating the human immune system to fight off infections, prevent allergic disease, and avoid malignancies. Together these eight reviews provide a background picture of DOCK8 immunodeficiency and related disorders, and identify areas for future research.

\section{Acknowledgements}

This work was supported by the Intramural Research Program of the NIH, NIAID. 


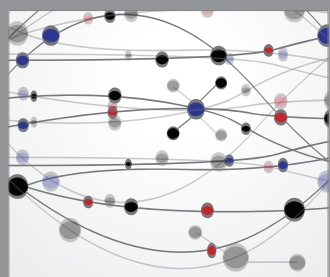

The Scientific World Journal
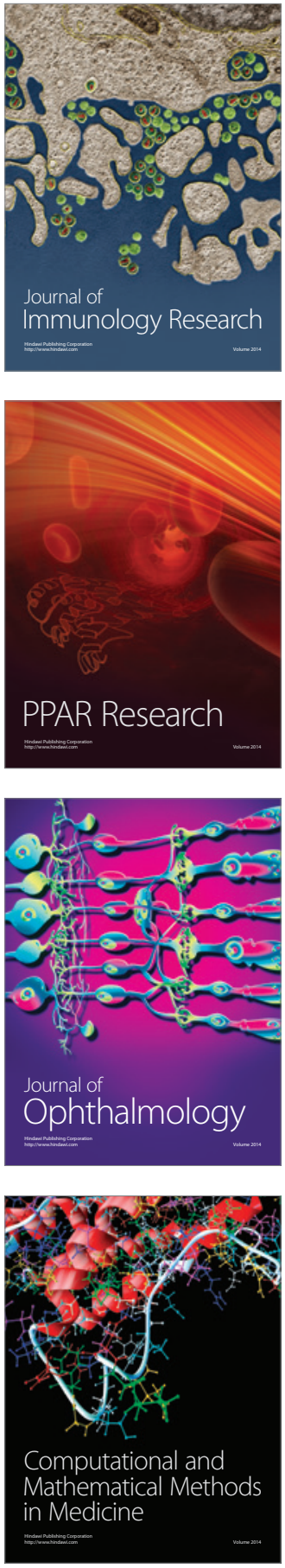

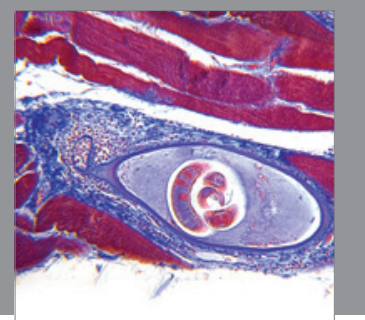

Gastroenterology

Research and Practice
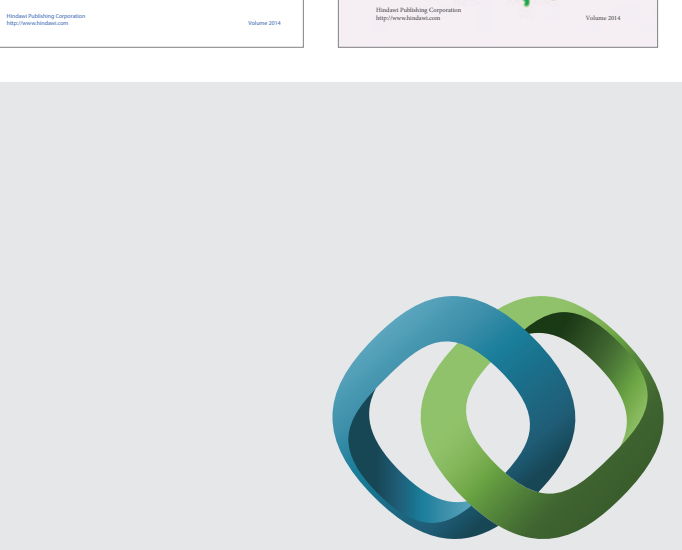

\section{Hindawi}

Submit your manuscripts at

http://www.hindawi.com
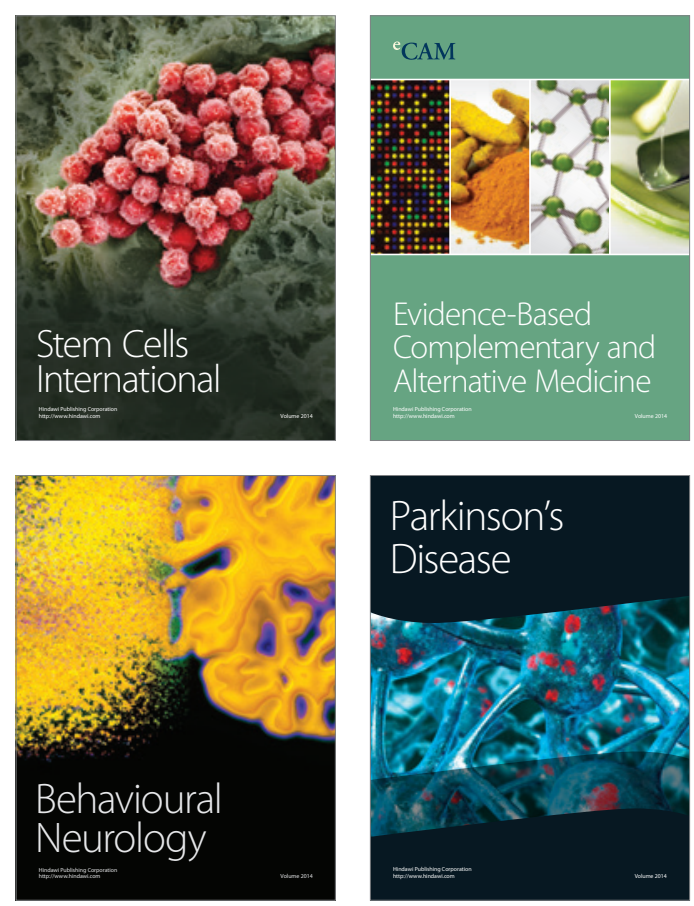

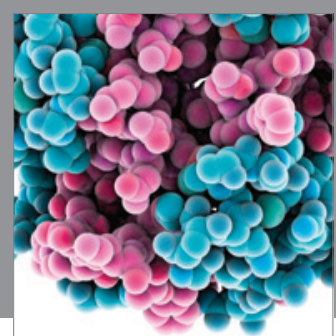

Journal of
Diabetes Research

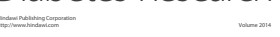

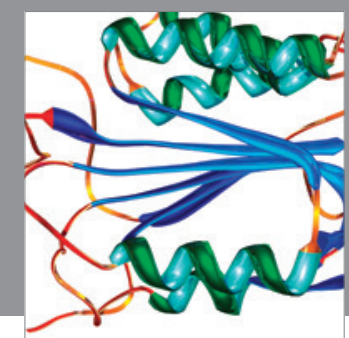

Disease Markers
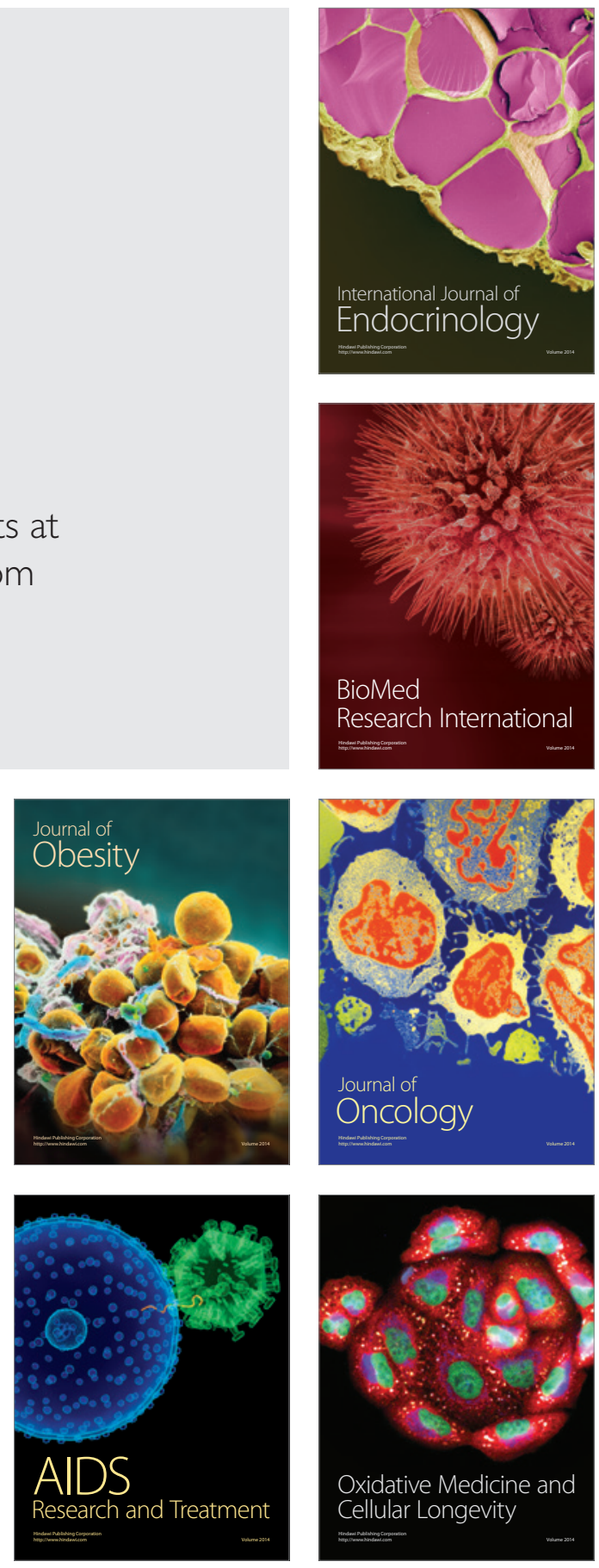